\title{
Power threshold and saturation of parametric decay instabilities near the upper hybrid resonance in plasmas
}

Hansen, S. K.; Nielsen, S. K.; Stober, J.; Rasmussen, J.; Salewski, Mirko; Stejner, M.; ASDEX Upgrade Team

Published in:

Physics of Plasmas

Link to article, DOI:

$10.1063 / 1.5091659$

Publication date:

2019

Document Version

Publisher's PDF, also known as Version of record

Link back to DTU Orbit

Citation (APA):

Hansen, S. K., Nielsen, S. K., Stober, J., Rasmussen, J., Salewski, M., Stejner, M., \& ASDEX Upgrade Team (2019). Power threshold and saturation of parametric decay instabilities near the upper hybrid resonance in plasmas. Physics of Plasmas, 26(6), [062102]. https://doi.org/10.1063/1.5091659

\section{General rights}

Copyright and moral rights for the publications made accessible in the public portal are retained by the authors and/or other copyright owners and it is a condition of accessing publications that users recognise and abide by the legal requirements associated with these rights.

- Users may download and print one copy of any publication from the public portal for the purpose of private study or research.

- You may not further distribute the material or use it for any profit-making activity or commercial gain

- You may freely distribute the URL identifying the publication in the public portal 


\section{Power threshold and saturation of parametric decay instabilities near the upper hybrid resonance in plasmas}

Cite as: Phys. Plasmas 26, 062102 (2019); https://doi.org/10.1063/1.5091659

Submitted: 04 February 2019. Accepted: 10 May 2019. Published Online: 04 June 2019

S. K. Hansen, S. K. Nielsen, J. Stober, J. Rasmussen (D), M. Salewski (D), M. Stejner (D), and ASDEX Upgrade Team

\section{ARTICLES YOU MAY BE INTERESTED IN}

Three-dimensional effect of particle motion on plasma filament dynamics

Physics of Plasmas 26, 062104 (2019); https://doi.org/10.1063/1.5093561

Study of Alfven eigenmodes stability in plasma with multiple NBI driven energetic particle species

Physics of Plasmas 26, 062502 (2019); https://doi.org/10.1063/1.5098347

Blob velocity scaling in diverted tokamaks: A comparison between theory and simulation

Physics of Plasmas 26, 032302 (2019); https://doi.org/10.1063/1.5080675

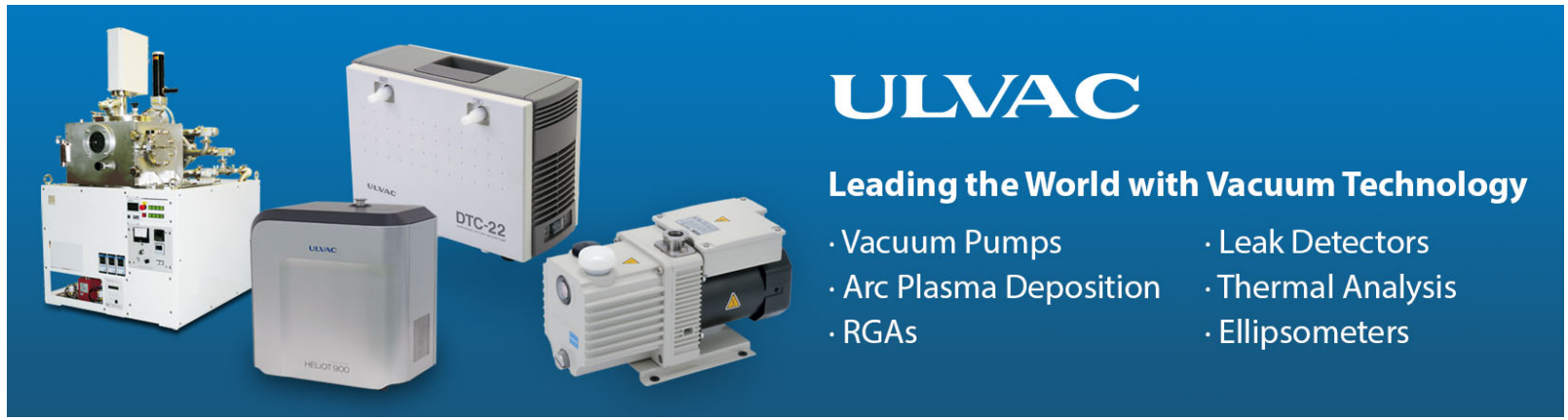




\title{
Power threshold and saturation of parametric decay instabilities near the upper hybrid resonance in plasmas
}

Cite as: Phys. Plasmas 26, 062102 (2019); doi: 10.1063/1.5091659

Submitted: 4 February 2019 • Accepted: 10 May 2019 •

Published Online: 4 June 2019

\author{
S. K. Hansen, ${ }^{1,2, a)}$ S. K. Nielsen, ${ }^{2}$ J. Stober, ${ }^{1}$ J. Rasmussen, $^{2}$ (D) M. Salewski, ${ }^{2}$ iD M. Stejner, ${ }^{2}$ \\ and ASDEX Upgrade Team
}

\author{
AFFILIATIONS \\ ${ }^{7}$ Max-Planck-Institut für Plasmaphysik, D-85748 Garching b. München, Germany \\ ${ }^{2}$ Department of Physics, Technical University of Denmark, DK-2800 Kgs. Lyngby, Denmark
}

a)Electronic mail: Soeren.Kjer.Hansen@ipp.mpg.de

\begin{abstract}
Parametric decay instabilities (PDIs) occur for large-amplitude waves in quadratically nonlinear media, where they provide a limit of validity of linear theories and allow efficient coupling between different, well-defined wave modes. We investigate PDIs near the upper hybrid resonance in plasmas by injection of high-power electron cyclotron (EC) waves at the ASDEX Upgrade tokamak. Our measurements of PDIs have an unprecedented frequency resolution, far below the ion cyclotron frequency, allowing the first observations of secondary and tertiary PDIs during the saturation phase in a controlled laboratory setting. Furthermore, we are for the first time able to systematically compare theoretical predictions of the EC wave power thresholds, which must be exceeded to excite such PDIs, with experimental observations, validating the theory. Our findings are relevant for EC wave heating and current drive in tokamaks and stellarators, including future fusion power plants, as well as in lowtemperature laboratory and industrial plasmas, inertial confinement fusion, and ionospheric modification experiments.
\end{abstract}

Published under license by AIP Publishing. https://doi.org/10.1063/1.5091659

\section{INTRODUCTION}

In a parametric decay instability (PDI), a large-amplitude pump wave decays to two daughter waves once its amplitude exceeds a nonlinear threshold. PDIs are ubiquitous in quadratically nonlinear media, including optical crystals, ${ }^{1}$ mechanical systems, ${ }^{2}$ fluids, ${ }^{3}$ and plasmas. ${ }^{4}$ The PDI threshold indicates the limit of validity of linear theories and should consequently not be exceeded in applications relying on a linear medium response. On the other hand, the nonlinear response above the PDI threshold allows efficient coupling of the pump wave to different, well-defined daughter wave modes, which is crucial for applications such as telecommunications ${ }^{1}$ and ionospheric modification experiments. ${ }^{5-11}$ It is thus of great importance to understand the PDI threshold, and the nonlinear response beyond it, to avoid the deleterious effects of an undesired nonlinear response and to exploit the nonlinear response for novel applications.

We consider PDIs occurring in connection with electron cyclotron resonance (ECR) heating of plasmas, which is widely applied for ionospheric modification experiments, ${ }^{5-11}$ as well as for generating and sustaining laboratory and industrial plasmas. ${ }^{12-14} \mathrm{~A}$ number of electromagnetic emission features in the electron cyclotron (EC) frequency range have been attributed to particular PDIs occurring in connection with ECR heating. As the dispersion relations of the involved waves are known, this has allowed investigation of various properties of the ionosphere, such as the magnetic field, ${ }^{6}$ conditions for electron acceleration, ${ }^{7}$ the electron temperature, ${ }^{8}$ the ion composition, ${ }^{9}$ and pump-generated plasma layers. ${ }^{10}$ PDIs in the EC frequency range have also been observed in a number of laboratory plasmas, including low-temperature experiments, ${ }^{15-18}$ inertial confinement fusion experiments, ${ }^{4}$ and magnetic confinement fusion experiments both in tokamaks ${ }^{19-26}$ and in stellarators. ${ }^{27-31}$

PDIs in laboratory plasmas have been used to demonstrate the occurrence of O-X-B heating, ${ }^{22,28,29}$ to provide direct heating, ${ }^{4,16-18,20,21,27,30}$ and can also deliver information about the plasma parameters ${ }^{15,19,23-26,31}$ but have generally been ignored when computing ECR heating and current drive characteristics. ${ }^{32}$ The occurrence of PDIs hampers, and may damage, laboratory EC wave diagnostics. ${ }^{23,24}$ Present theories even suggest that PDIs can lead to significantly different ECR heating and current drive characteristics than those expected from linear theories in some cases. ${ }^{33}$ The detailed study of PDIs in the EC frequency range of a laboratory plasma presented here is thus of 
significant interest, considering the increasing importance of ECR heating and current drive in present and future large-scale fusion experiments and the possibility of validating PDI models used for ionospheric modification experiments in laboratory plasmas, where the plasma parameters can be controlled and monitored to a much larger extent.

We specifically investigate PDIs occurring for X-mode polarized radiation near the upper hybrid resonance (UHR), where the pump wave frequency, $f_{0}$, equals $f_{U H}=\sqrt{f_{p e}^{2}+f_{c c}^{2}}, f_{p e}$ and $f_{c e}$ are the electron plasma and cyclotron frequencies, respectively. Strong enhancement of the electric field of the X-mode pump wave near the $\mathrm{UHR}^{26,34}$ allows the thresholds of various PDIs to be exceeded. PDIs for $\mathrm{X}$-mode radiation near the UHR are relevant for fundamental ECR heating both with the X-mode $e^{19-21,30,35}$ and the O-mode ${ }^{21,27}$ (O-mode can be converted to X-mode by reflections ${ }^{21,26,27}$ ) for $\mathrm{O}-\mathrm{X}-\mathrm{B}$ heating, ${ }^{22,28,29,36-38}$ for collective Thomson scattering, ${ }^{24-26,31}$ and for ionospheric modification experiments; ${ }^{39}$ similar PDIs also enter as secondary instabilities in the saturation phase of two-plasmon decay instabilities occurring for harmonic X-mode ECR heating ${ }^{23,33}$ and direct-drive inertial confinement fusion. ${ }^{4,40}$ Particular cases where such PDIs may occur in future experiments include ECR start-up at ITER, MAST-U, NSTX-U, and future fusion reactors, ${ }^{41-43} \mathrm{O}-\mathrm{X}-\mathrm{B}$ heating at $\mathrm{W} 7-\mathrm{X}$, and ionospheric modification experiments with significant $\mathrm{O}-\mathrm{X}$-conversion at the $\mathrm{O}$-mode cutoff.

Energy conservation in the decay process imposes the selection rule that the daughter wave frequencies, $f_{1,2}$, should add up to the pump frequency, i.e., $f_{1}+f_{2}=f_{0}$. A number of theories have predicted the PDI near the UHR with the lowest threshold to be decay of the X-mode pump wave to a low-frequency lower hybrid (LH) wave and a high-frequency electron Bernstein wave (EBW); ${ }^{26,35-37,39}$ the LH wave frequency is $f_{1} \sim f_{L H} \ll f_{p e}$, where $f_{L H}$ is the cold LH frequency and the EBW frequency is $f_{2}=f_{0}-f_{1} \approx f_{U H}-f_{L H}$. The occurrence of this instability is corroborated by experiments ${ }^{19-22,24-29,31}$ and simulations. $38,44,45$

Here, we report an experiment from the ASDEX Upgrade (AUG) tokamak which allows the first systematic comparison between experimental observations and theoretical predictions ${ }^{26}$ of the threshold of the above PDI. The unprecedented frequency resolution of the reported experiment additionally allows the first observations of the fine structure of spectra excited by PDIs near the UHR in a controlled laboratory setting. The fine structure consists of variations on the order of the ion cyclotron frequency, $f_{c i} \ll f_{L H}$, and is crucial for describing secondary and tertiary PDIs, involving coupling of the daughter waves excited by the primary instability or the pump wave itself to EBWs and ion Bernstein waves (IBWs), which eventually lead to saturation.

This paper is arranged as follows: Sec. II describes the experimental setup, Sec. III presents the experimental results, Sec. IV discusses the interpretation of the experimental results, and, finally, Sec. V provides our conclusions.

\section{EXPERIMENTAL SETUP}

A schematic of the experimental setup is shown in Fig. 1. In the experiment, $105 \mathrm{GHz} \mathrm{O}$-mode radiation is injected from the low-field side of AUG at a toroidal angle of $-4.8^{\circ}$, using a gyrotron from the system described in Ref. 46. The O-mode fraction of the scattered radiation near $105 \mathrm{GHz}$ is picked up by a steerable radiometer on the low-field side, which includes a fast acquisition system ${ }^{47}$ capable of

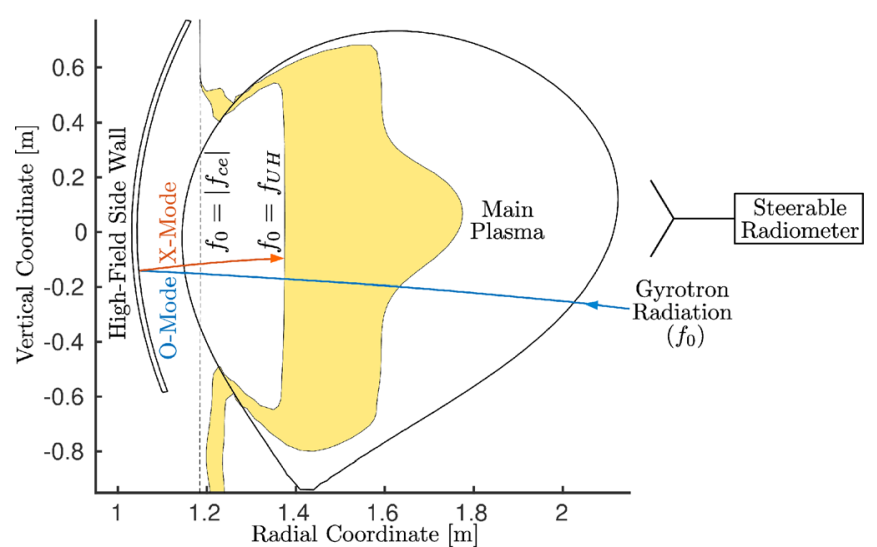

FIG. 1. Schematic of the experimental setup in AUG discharge 34575. No propagating X-mode exists in the shaded region.

resolving the fine structure of the PDI spectra. The ECR $\left(f_{0}=\left|f_{c e}\right|\right)$ of the $105 \mathrm{GHz}$ radiation is located far on the high-field side and is optically thin for O-mode radiation. A significant fraction of the incident radiation can thus reflect off the high-field side wall and re-enter the plasma in the X-mode. When the ECR is not optically thick for the $\mathrm{X}$-mode radiation, the electric field near the UHR may become sufficiently large to excite PDIs. The experimental setup is similar to the ones used for fundamental O-mode ECR heating, ${ }^{20,21,27}$ collective Thomson scattering, ${ }^{24-26,31}$ and EBW start-up; ${ }^{41-43}$ it also has much in common with the setups used for O-X-B heating ${ }^{22,28,29}$ and ionospheric modification experiments, ${ }^{5-11}$ the main difference being that $\mathrm{O}$-X-conversion occurs at the O-mode cutoff $\left(f_{0}=f_{p e}\right)$ in these setups.

The analyzed experiment is an $\mathrm{H}$-mode, ${ }^{48}$ deuterium discharge during which the toroidal magnetic field, $B_{t}$, is swept from $-2.66 \mathrm{~T}$ to $-2.43 \mathrm{~T}$ over $3.5 \mathrm{~s}$. For us, the most important effect of the sweep is the displacement of the ECR toward the high-field side, reducing its absorption by an order of magnitude. This is seen in Fig. 2; the spread in the data is due to uncertainties in the experimental equilibria.

To assess the development of the PDIs during the $B_{t}$-sweep, 200 evenly spaced $2 \mathrm{~ms}$ pulses are generated by the gyrotron. For a comparison of the experimental and theoretical ${ }^{26} \mathrm{PDI}$ thresholds, the gyrotron power, $P_{0}$, is varied from 0 to $300 \mathrm{~kW}$ during each pulse. The variation of $P_{0}$ during a pulse is seen in Fig. 3. The scattered signal is recorded in $1 \mathrm{~ms}$ measurement pulses around the center of each gyrotron pulse. Power spectra are obtained by performing fast Fourier transforms on 655 ns windows of the recorded signal for all measurement pulses, giving a frequency resolution of $1.53 \mathrm{MHz}$, well below $f_{c i}$ of deuterium in the experiment $(\sim 30 \mathrm{MHz})$.

\section{EXPERIMENTAL RESULTS}

The mean spectra recorded during three measurement pulses at different points in the $B_{t}$-sweep are seen in Fig. 4 . All spectra are taken from the part of the $B_{t}$-sweep where PDI peaks are consistently visible and illustrate the qualitative development of these peaks. The gyrotron line is visible slightly below $105 \mathrm{GHz}\left(f_{0}=104.93 \mathrm{GHz}\right)$ in all spectra. The smaller peaks, separated by $0.7-1.1 \mathrm{GHz}$ (slightly more than $f_{L H}$ at the UHR) from the gyrotron peak, originate from the daughter waves excited by PDIs; the mechanisms through which they are generated are discussed in Sec. IV. As seen in Fig. 4, the PDI peaks start out as a 


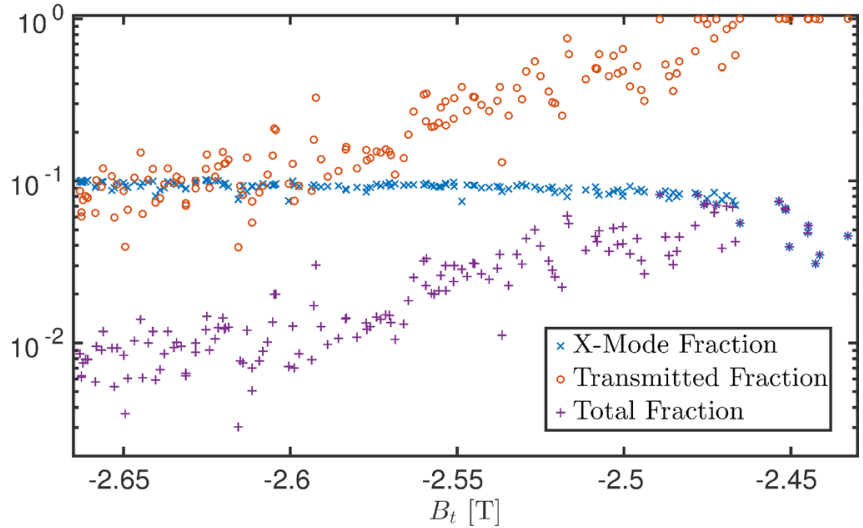

FIG. 2. Fraction of reflected power coupled to the plasma in X-mode $(\times)$, fraction of injected power not absorbed at the ECR $\left({ }^{\circ}\right)$, and total fraction of injected power reaching the UHR in X-mode (+) vs $B_{t}$ in AUG discharge 34575 , based on the theory of Ref. 26.

few well-defined lines in pulse (a) and develop an increasingly complex structure, visible in pulses (b) and (c), as $B_{t}$ is swept. The fine structure in pulses (b) and (c) indicates that secondary and tertiary PDIs occur during the saturation phase. In pulse (b), peaks separated by approximately $50 \mathrm{MHz}$ (roughly $2 f_{c i}$ at the UHR) occur around the primary peaks, indicating decay of the primary daughter waves to EBWs and second-order IBWs as the secondary PDI. In pulse (c), additional peaks separated by approximately $25 \mathrm{MHz}$ (roughly $f_{c i}$ at the UHR) appear throughout the frequency range covered by the radiometer, indicating decay of the pump wave to EBWs and arbitraryorder IBWs as the tertiary PDI. For reference, we plot the theoretical frequency of the most unstable up- and down-shifted primary daughter waves from Ref. 26, along with $f_{0} \pm f_{L H}$, in Fig. 4. The theoretical frequency shift represents an upper bound on the true shift, while $f_{L H}$ represents a lower bound. This may be explained by use of the dipole approximation in Ref. 26 and finite temperature effects, respectively.

To extract information about the $P_{0}$-dependence of the PDI peaks, we calculate the spectral power, $S$, in the frequency ranges 103.8-104.2 GHz and 105.6-106.0 GHz for all spectra obtained during

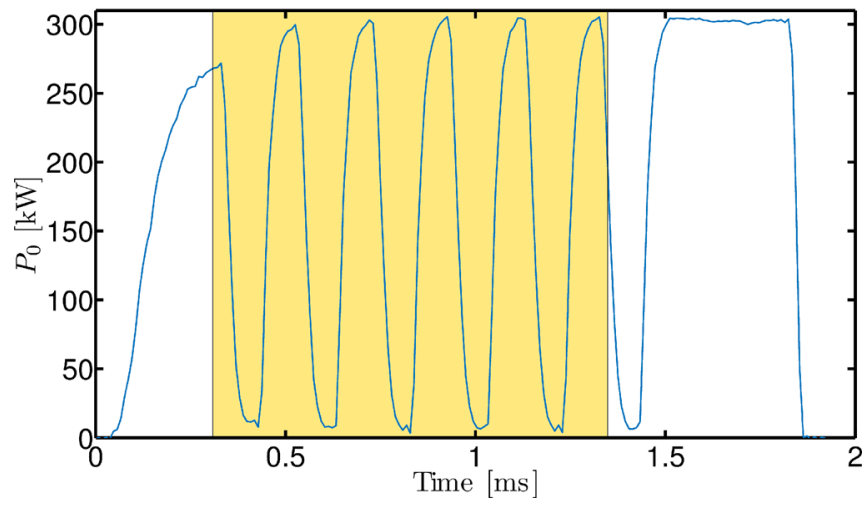

FIG. 3. Modulation of $P_{0}$ during a gyrotron pulse. The spectrum of the scattered radiation is measured using the steerable radiometer in the shaded interval during all gyrotron pulses.

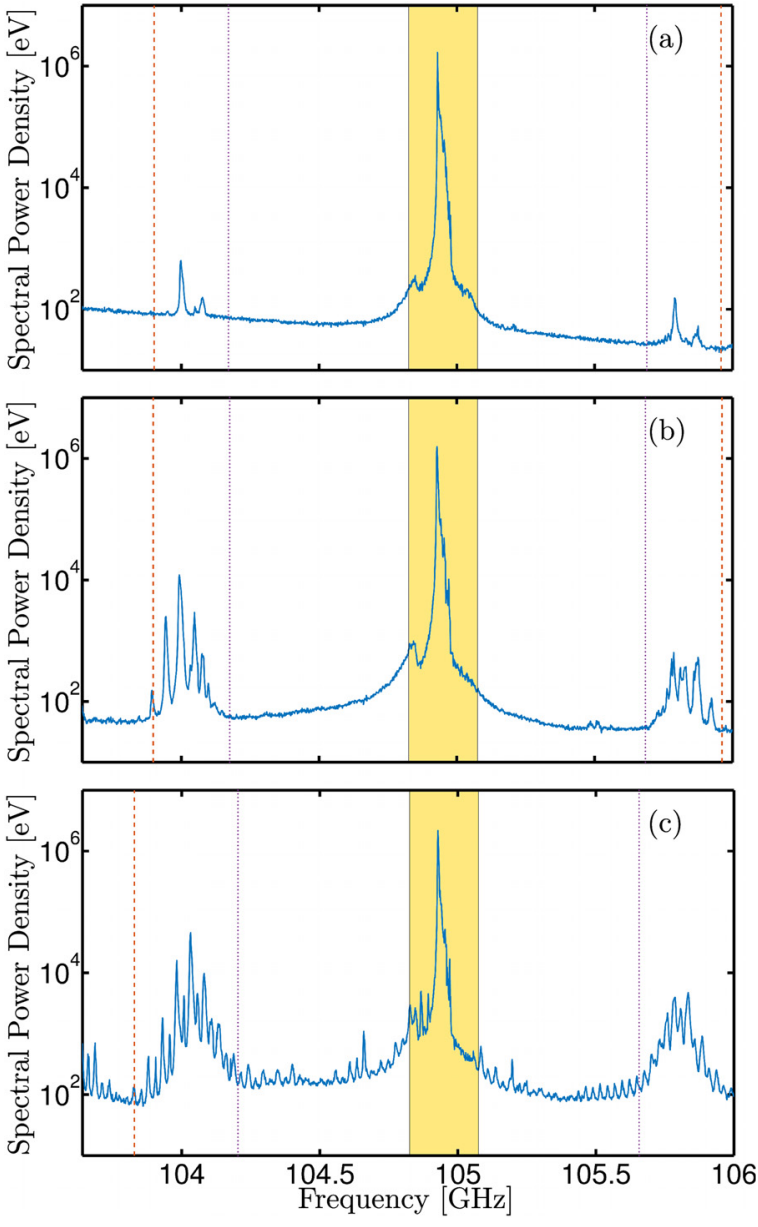

FIG. 4. Mean spectra recorded by the steerable radiometer in AUG discharge 34575 at $B_{t}=-2.53 \mathrm{~T}(\mathrm{a}), B_{t}=-2.48 \mathrm{~T}(\mathrm{~b})$, and $B_{t}=-2.44 \mathrm{~T}$ (c). The shaded areas indicate the stopband of the notch filter near $f_{0}$, where the calibration is uncertain. The dashed lines indicate the most unstable modes according to Ref. 26; the dotted lines indicate $f_{0} \pm f_{L H}$.

a measurement pulse. The frequency ranges are chosen such that they contain the primary PDI peaks, but not the gyrotron peak, and changing their precise limits does not change the subsequent conclusions if this remains the case. We derive $S\left(P_{0}\right)$ using $P_{0}$-waveforms similar to that in Fig. 3. Figure 5 shows $S$ vs $P_{0}$ for the measurement pulses whose mean spectra were shown in Fig. 4. Evidently, $S$ depends nonlinearly on $P_{0}$, as expected for PDI-generated peaks. In pulse (a), $S$ only deviates appreciably from the background level for $P_{0}>250 \mathrm{~kW}$ while in pulses (b) and (c), $S$ increases roughly linearly with $P_{0}$ until $100 \mathrm{~kW}$ and $50 \mathrm{~kW}$, respectively, but supra-linearly beyond these values. We note that $S$ saturates for $P_{0}>200 \mathrm{~kW}$ in pulse (b), while in pulse (c), some saturation occurs for $P_{0} \in[100 \mathrm{~kW}, 250 \mathrm{~kW}]$, followed by more rapid growth for $P_{0}>250 \mathrm{~kW}$. This corroborates that the secondary instability involves the primary daughter waves, stabilizing $S$ in the PDI region, while the tertiary instability involves the pump wave, opening additional channels through which power can flow into the PDI region, as stated above. 

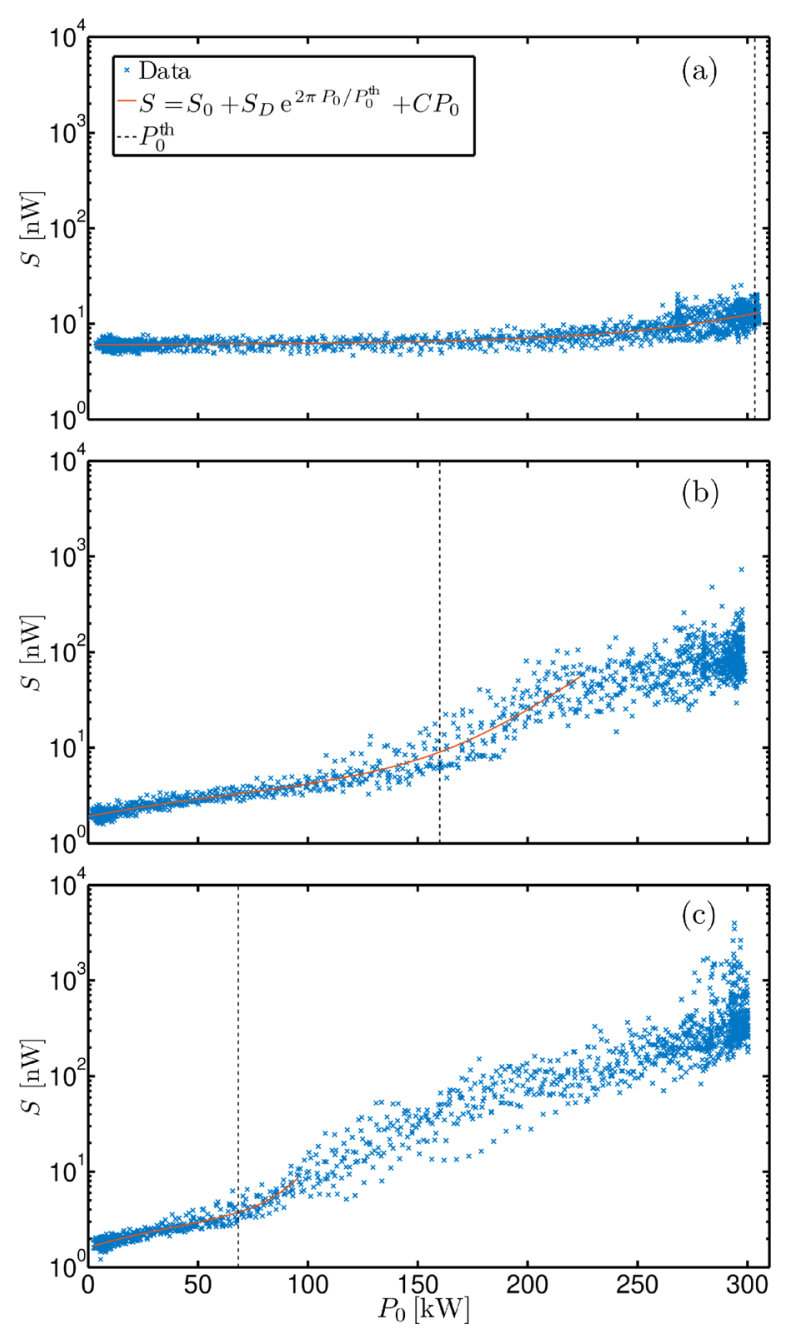

FIG. 5. $S$ vs $P_{0}$ in AUG discharge 34575 at $B_{t}=-2.53 \mathrm{~T}(\mathrm{a}), B_{t}=-2.48 \mathrm{~T}$ (b), and $B_{t}=-2.44 \mathrm{~T}$ (c). The experimental data $(\times)$ are fitted using Eq. (1); the fits are marked by solid lines. The dashed lines indicate the fitted $P_{0}^{\text {th }}$-values.

To compare the experimental $S\left(P_{0}\right)$ with the dependence expected from Ref. 26, we introduce a physical model. First, $S$ contains a thermal background contribution, $S_{0}$, independent of $P_{0}$. Second, $S$ contains a $P_{0}$-dependent contribution of the form $S_{D} \mathrm{e}^{2 \pi P_{0} / P_{0}^{\text {th }}}$ from the waves amplified by the primary PDI; $S_{D}$ is the thermal background signal due to these waves, $\mathrm{e}^{2 \pi P_{0} / P_{0}^{\mathrm{th}}}$ is the convective PDI amplification factor in the absence of collisions and saturation, and $P_{0}^{\text {th }}$ is the convective PDI threshold defined in Refs. 49-51. Finally, $S$ contains a contribution linear in $P_{0}$, written as $C P_{0}$, due to collective Thomson scattering of the gyrotron radiation ${ }^{24,52}$ and increased emission due to absorption at the ECR. Addition of the contributions yields

$$
S=S_{0}+S_{D} \mathrm{e}^{2 \pi P_{0} / P_{0}^{\mathrm{th}}}+C P_{0} .
$$

Least-square fits of the logarithm of the measured $S$ to the logarithm of Eq. (1) are shown in Fig. 5. In the fits, we require all parameters to be non-negative and only include data up to a $P_{0}$-value where

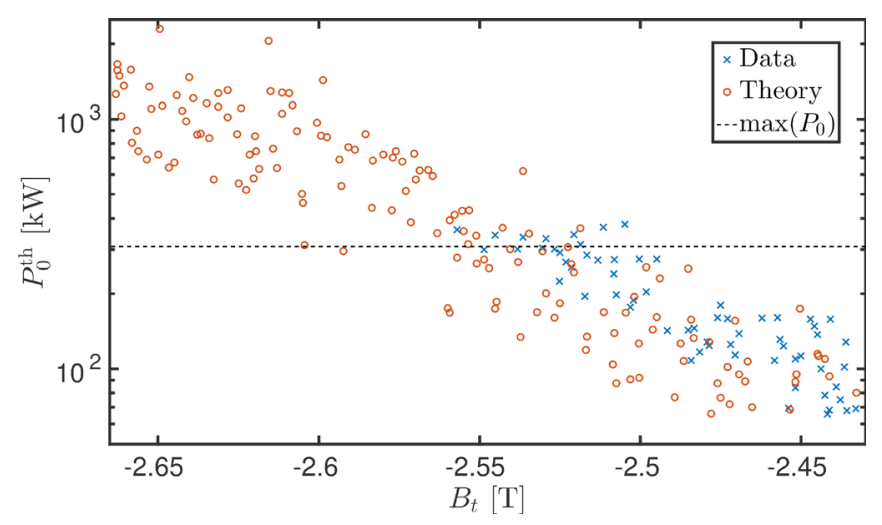

FIG. 6. Experimental $(\times)$ and theoretical $\left({ }^{\circ}\right) P_{0}^{\text {th }}$ vs $B_{t}$ in AUG discharge 34575 . The dashed line marks $\max \left(P_{0}\right)$ in the experiment.

saturation sets in, as this effect is not included in the model. The cutoff power used is $1.4 P_{0}^{\text {th }}$, provided that this value is smaller than $\max \left(P_{0}\right)$, and represents a rough experimental estimate of the gyrotron power threshold of the secondary instability.

To facilitate a comparison with theory, ${ }^{26}$ the fitted $P_{0}^{\text {th }}$-values are indicated in Fig. 5. As expected, they are close to the points where the supra-linear increase in $S$ with $P_{0}$ begins. Figure 6 shows the experimental values of $P_{0}^{\text {th }}$, obtained from fits similar to those in Fig. 5, and the theoretical predictions as functions of $B_{t}$. Note that we have only included fitted $P_{0}^{\text {th }}$-values from measurement pulses with a clear nonlinear phase and that we have only included theoretical $P_{0}^{\text {th }}$-values for equilibria where the UHR was reached by the reflected X-mode ray. The theoretical $P_{0}^{\text {th }}$-values show a spread due to uncertainties in the experimental equilibria used for their calculation. The experimental $P_{0}^{\text {th }}$-values lie within this spread throughout the $B_{t^{-}}$sweep, albeit with a small bias toward higher $P_{0}^{\text {th }}$-values. We also remark that PDI peaks are only observed for $\left|B_{t}\right|<2.56 \mathrm{~T}$ in the experiment, which coincides closely with the point where the theoretical $P_{0}^{\text {th }}$ drops below $\max \left(P_{0}\right)$, as seen in Fig. 6.

\section{DISCUSSION}

Having presented the main results of the experiment, we now discuss their interpretation in more detail. First of all, the waves excited by the PDIs under consideration are electrostatic in nature, meaning that they cannot be detected directly by the steerable radiometer used in the experiment. More specifically, the waves must somehow give rise to O-mode waves propagating toward the detector, owing to the cutoff region of the X-mode radiation (see Fig. 1) and the settings of the steerable radiometer polarizers.

For the primary PDI, a feasible mechanism is always provided by mixing of the injected waves with the low-frequency LH daughter waves excited by the PDI, which should lead to the observed downand up-shifted peaks at the beat frequencies $\left(f_{0} \mp f_{1}\right)$. As long as the power of the injected waves remains large compared with that of the daughter waves, which should at least hold until saturation sets in, this signal will be proportional to the daughter wave power and thus provide a reliable measure of the PDI threshold. An alternative mechanism is provided by linear conversion of the high-frequency daughter waves to $\mathrm{O}$-mode radiation. The efficiency of this mechanism is highly dependent on the direction of propagation of the high-frequency 
daughter waves, as EBWs undergo linear conversion to X-mode waves at the UHR. ${ }^{29}$ These X-mode waves may then be converted to Omode waves by a wall reflection. On the other hand, EBWs going directly to the ECR will be almost completely absorbed for all reasonable conditions. ${ }^{29}$ As the EBWs are backward propagating under the conditions in AUG $\left(f_{p e}<\sqrt{3}\left|f_{c e}\right|\right),{ }^{26}$ high-frequency waves which are forwardscattered with respect to the pump wave vector will go directly to the ECR, while backscattered waves may undergo the conversion process described above. As the theory of Ref. 26 is based on the dipole approximation, which ignores the pump wave vector relative to those of the daughter waves, it does not provide direct information about the direction in which the high-frequency daughter waves propagate. However, the fact that the observed frequency shift of the daughter waves relative to the pump wave is smaller than what is expected theoretically (see Fig. 4) indicates forward scattering, as already noted in Ref. 26; this is also in agreement with recent numerical simulations. ${ }^{45}$ Within the 1D-picture presented here, it thus seems that linear conversion of the high-frequency daughter waves to O-mode waves will not be efficient. This may additionally explain why the observed signal only rises two orders of magnitude above the linear background even at the highest power levels in the cases with the lowest ECR absorption.

For the secondary PDI, we postulated decay of the primary daughter waves into secondary EBWs and second-order IBWs, based on the observation of peaks separated by approximately $2 f_{c i}$ around the primary PDI peaks. While we have not explicitly computed the threshold for this process, we have confirmed the possibility of satisfying the selection rules when the secondary EBW is forwardscattered with respect to the primary one; the details are given in the Appendix. The main mechanism through which O-mode radiation may be generated by this instability is mixing of the waves near the primary daughter frequencies $\left(f_{0} \mp f_{1}\right)$ with the second-order IBWs, generating waves at approximately $f_{0} \mp f_{1} \mp 2 n f_{c i}$, where $n \in \mathbb{N}$. For the tertiary instability, similar mixing involving the injected waves and arbitraryorder IBWs is expected to explain the generation of O-mode radiation.

We note that there are still several points deserving further investigation. First, it would be of interest to extend the theory of Ref. 26 beyond the dipole approximation. This would allow us to obtain theoretical frequency shifts closer to the experimental values (see Fig. 4), as well as to investigate the directions of propagation of the daughter waves and the possibility of absolute PDIs, e.g., considered by Ref. 36, in greater detail. Second, the secondary and tertiary PDIs indicated by the experiment should be investigated theoretically. From this, we could obtain secondary and tertiary PDI thresholds for comparison with the experimental observations and construct a model describing the saturation phase of PDIs near the UHR. Finally, the experiment itself could be improved by investigating the low-frequency waves excited by the PDIs directly. Future experiments at AUG will be capable of this, using a B-dot probe ${ }^{53}$ connected to the second channel of the fast acquisition system employed in the present Paper. ${ }^{47}$

\section{CONCLUSION}

We have performed the first experimental investigation of PDIs near the UHR in a laboratory plasma capable of resolving the fine structure of the spectra excited by the PDIs and providing a comparison of the pump power necessary to excite the primary instability with the theory.
The recorded spectra showed initial development of PDI peaks separated by slightly more than $f_{L H}$ from the pump peak, followed by development of peaks separated by $2 f_{c i}$ from the primary PDI peaks, and finally development of peaks separated by $f_{c i}$ throughout the frequency region covered by the radiometer. This indicates initial decay of the pump wave to EBWs and LH waves, followed by decay of the primary EBWs to other EBWs and second-order IBWs, and finally decay of the pump wave to EBWs and arbitraryorder IBWs.

By modulating the gyrotron power, we were able to obtain experimental PDI thresholds which corroborated the theoretical predictions from Ref. 26. These findings indicate that the possibility of PDIs occurring near the UHR during ECR start-up at ITER, MAST-U, NSTX-U, and future fusion reactors may be assessed using existing theories. The theory tested here is also applicable to O-X-B heating, planned for W7-X, and ionospheric modification experiments with significant $\mathrm{O}-$ $\mathrm{X}$-conversion at the $\mathrm{O}$-mode cutoff, provided that the plasma is only moderately overdense, $f_{p e}<\sqrt{3}\left|f_{c e}\right|$, at the UHR. For $f_{p e}>\sqrt{3}\left|f_{c e}\right|$, the theory of Ref. 36 is applicable.

\section{ACKNOWLEDGMENTS}

This work was supported by a research Grant (15483) from VILLUM FONDEN. This work has been carried out within the framework of the EUROfusion Consortium and has received funding from the Euratom research and training program 2014-2018 and 2019-2020 under Grant Agreement No. 633053. The views and opinions expressed herein do not necessarily reflect those of the European Commission.

\section{APPENDIX: THE SECONDARY INSTABILITY}

Here, we show the possibility of satisfying the selection rules for decay of a primary EBW daughter into secondary EBW and second-order IBW daughters near the UHR when $f_{p e}<\sqrt{3}\left|f_{c e}\right|$. We use the EBW dispersion relation from Ref. 26 for propagation perpendicular to the background magnetic field

$$
f_{2}=f_{U H}-\frac{f_{p e}^{2}}{2 f_{U H}} \frac{r_{L e}^{2} k_{2}^{2}}{1-f_{p e}^{2} /\left(3 f_{c e}^{2}\right)}, \quad f_{2}^{\prime}=f_{U H}-\frac{f_{p e}^{2}}{2 f_{U H}} \frac{r_{L e}^{2}\left(k_{2}^{\prime}\right)^{2}}{1-f_{p e}^{2} /\left(3 f_{c e}^{2}\right)},
$$

where $f_{2}$ and $f_{2}^{\prime}$ are the primary and secondary EBW frequencies, $k_{2}$ and $k_{2}^{\prime}$ are the primary and secondary EBW wave numbers, $r_{L e}$ is the thermal electron Larmor radius defined in Ref. 26, and we have expanded the dispersion relation from Ref. 26 to first order in $r_{L e}^{2} k_{2}^{2} \ll 1$ and $r_{L e}^{2}\left(k_{2}^{\prime}\right)^{2} \ll 1$. Using the frequency selection rule of the primary PDI, $f_{2}=f_{0}-f_{1} \approx f_{U H}-f_{L H}$, we obtain

$$
\left|k_{2}\right| \approx \frac{\sqrt{2 f_{L H} f_{U H}\left[1-f_{p e}^{2} /\left(3 f_{c e}^{2}\right)\right]}}{f_{p e} r_{L e}},
$$

which does indeed satisfy $r_{L e}^{2} k_{2}^{2} \ll 1$ for the usual ordering $\left(f_{L H} \ll f_{p e} \lesssim f_{U H}\right)$. The dispersion relation of the second-order IBW for propagation perpendicular to the background magnetic field in a simple plasma is given by Eq. (4.275) of Ref. 54, 


$$
f_{1}^{\prime}=2 f_{c i}\left[1-\frac{3 r_{L i}^{2}\left(k_{1}^{\prime}\right)^{2}}{8\left(1-3 v_{A}^{2} / c^{2}\right)}\right],
$$

where $f_{1}^{\prime}$ is the IBW frequency, $k_{1}^{\prime}$ is the IBW wave number, $r_{L i}$ is the thermal ion Larmor radius, $c$ is the vacuum speed of light, $v_{A}=c / \sqrt{1+f_{p i}^{2} / f_{c i}^{2}} \ll c$ (since the ion plasma frequency, $\left.f_{p i}>f_{L H} \gg f_{c i}\right)$ is the Alfvén speed, and the expression is valid to first order in $r_{L i}^{2}\left(k_{1}^{\prime}\right)^{2} \ll 1$. Note that generally $r_{L i} \gg r_{L e}$, so $\left|k_{1}^{\prime}\right|$ should be significantly smaller than $\left|k_{2}\right|$ and $\left|k_{2}^{\prime}\right|$. Now, using the frequency selection rule of the secondary PDI, $f_{2}^{\prime}=f_{2}-f_{1}^{\prime} \approx f_{U H}$ $-f_{L H}-2 f_{c i}$, Eq. (A1) yields

$$
\left|k_{2}^{\prime}\right| \approx \frac{\sqrt{2\left(f_{L H}+2 f_{c i}\right) f_{U H}\left[1-f_{p e}^{2} /\left(3 f_{c e}^{2}\right)\right]}}{f_{p e} r_{L e}} \approx\left|k_{2}\right|\left(1+\frac{f_{c i}}{f_{L H}}\right) ;
$$

the last approximation follows by expansion to first order in $f_{c i} / f_{L H} \ll 1$. Taking $\mathbf{k}_{2}$ and $\mathbf{k}_{2}^{\prime}$ to point in the same direction, the wave vector selection rule ${ }^{26}$ of the secondary PDI gives

$$
k_{1}^{\prime}=k_{2}-k_{2}^{\prime} \approx-\frac{f_{c i}}{f_{L H}} k_{2},
$$

with the negative sign indicating that $\mathbf{k}_{1}^{\prime}$ points in the opposite direction of $\mathbf{k}_{2}$ and $\mathbf{k}_{2}^{\prime}$. Plugging Eq. (A2) into Eq. (A5), we find

$$
\begin{aligned}
r_{L i}^{2}\left(k_{1}^{\prime}\right)^{2} & \approx \frac{2 f_{c i}^{2} f_{U H}\left[1-f_{p e}^{2} /\left(3 f_{c e}^{2}\right)\right]}{f_{L H} f_{p e}^{2}} \frac{r_{L i}^{2}}{r_{L e}^{2}} \\
& =2 \sqrt{\frac{Z_{i} m_{e}}{m_{i}}} \frac{T_{i}}{Z_{i} T_{e}} \frac{\left|f_{c e}\right| f_{U H}^{2}}{f_{p e}^{3}}\left(1-\frac{f_{p e}^{2}}{3 f_{c e}^{2}}\right),
\end{aligned}
$$

where $Z_{i}$ is the ion charge number, $m_{e}$ and $m_{i}$ are the electron and ion masses, and $T_{e}$ and $T_{i}$ are the electron and ion temperatures. If $f_{p e}$ and $\left|f_{c e}\right|$ have similar orders of magnitude, $r_{L i}^{2}\left(k_{1}^{\prime}\right)^{2}$ $\sim \sqrt{Z_{i} m_{e} / m_{i}}\left[T_{i} /\left(Z_{i} T_{e}\right)\right] \ll 1$, as assumed in Eq. (A3). Particularly, typical conditions near the UHR of $105 \mathrm{GHz}$ radiation in AUG discharge 34575 are $Z_{i}=1, m_{i} / m_{e}=3.67 \times 10^{3}$, electron density $\approx 3.1 \times 10^{19} \mathrm{~m}^{-3}$, background magnetic field $\approx 3.3 \mathrm{~T}$, and $T_{e} \approx T_{i}$, giving $r_{L e}^{2} k_{2}^{2} \approx 0.055, r_{L e}^{2}\left(k_{2}^{\prime}\right)^{2} \approx 0.059$, and $r_{L i}^{2}\left(k_{1}^{\prime}\right)^{2} \approx 0.24$. It is thus possible to satisfy the selection rules of the secondary PDI in the region of validity of the dispersion relations.

\section{REFERENCES}

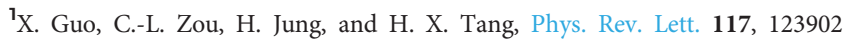
(2016).

${ }^{2}$ F. Brau, H. Vandeparre, A. Sabbah, C. Poulard, A. Boudaoud, and P. Damman, Nat. Phys. 7, 56 (2011).

${ }^{3}$ Q. Aubourg and N. Mordant, Phys. Rev. Lett. 114, 144501 (2015).

${ }^{4}$ D. H. Froula, B. Yaakobi, S. X. Hu, P.-Y. Chang, R. S. Craxton, D. H. Edgell, R. Follett, D. T. Michel, J. F. Myatt, W. Seka, R. W. Short, A. Solodov, and C. Stoeckl, Phys. Rev. Lett. 108, 165003 (2012).

${ }^{5}$ B. Thidé, H. Kopka, and P. Stubbe, Phys. Rev. Lett. 49, 1561 (1982).

${ }^{6}$ T. B. Leyser, B. Thidé, H. Derblom, Å. Hedberg, B. Lundborg, P. Stubbe, and H. Kopka, Phys. Rev. Lett. 63, 1145 (1989).

${ }^{7}$ T. B. Leyser, Space Sci. Rev. 98, 223 (2001)

${ }^{8}$ P. A. Bernhardt, C. A. Selcher, R. H. Lehmberg, S. Rodriguez, J. Thomason, M. McCarrick, and G. Frazer, Ann. Geophys. 27, 4409 (2009).
${ }^{9}$ P. A. Bernhardt, C. A. Selcher, R. H. Lehmberg, S. P. Rodriguez, J. F. Thomason, K. M. Groves, M. J. McCarrick, and G. J. Frazer, Phys. Rev. Lett. 104, 165004 (2010).

${ }^{10}$ E. Sergeev, S. Grach, A. Shindin, E. Mishin, P. Bernhardt, S. Briczinski, B. Isham, M. Broughton, J. LaBelle, and B. Watkins, Phys. Rev. Lett. 110, 065002 (2013).

${ }^{11}$ C. J. Bryers, M. J. Kosch, A. Senior, M. T. Rietveld, and T. K. Yeoman, J. Geophys. Res. Space Phys. 118, 7472, https://doi.org/10.1002/2013JA019429 (2013).

${ }^{12}$ J. Asmussen, J. Vac. Sci. Technol. A 7, 883 (1989).

${ }^{13}$ V. Erckmann and U. Gasparino, Plasma Phys. Controlled Fusion 36, 1869 (1994).

${ }^{14}$ R. Geller, Electron Cyclotron Resonance Ion Sources and ECR Plasmas (IOP Publishing, Bristol, 1996).

${ }^{15}$ S. Hiroe and H. Ikegami, Phys. Rev. Lett. 19, 1414 (1967).

${ }^{16}$ R. P. H. Chang, M. Porkolab, and B. Grek, Phys. Rev. Lett. 28, 206 (1972).

${ }^{17}$ B. Grek and M. Porkolab, Phys. Rev. Lett. 30, 836 (1973).

${ }^{18}$ M. Okabayashi, K. Chen, and M. Porkolab, Phys. Rev. Lett. 31, 1113 (1973).

${ }^{19}$ F. S. McDermott, G. Bekefi, K. E. Hackett, J. S. Levine, and M. Porkolab, Phys. Fluids 25, 1488 (1982).

${ }^{20}$ D. G. Bulyginskii, V. V. D’yachenko, M. A. Irzak, M. M. Larionov, L. S. Levin, G. A. Serebrenyi, and N. V. Shustova, Sov. J. Plasma Phys. 12, 77 (1986).

${ }^{21}$ Z. A. Pietrzyk, A. Pochelon, R. Behn, A. Bondeson, M. Dutch, T. P. Goodman, M. Q. Tran, and D. R. Whaley, Nucl. Fusion 33, 197 (1993).

${ }^{22}$ V. Shevchenko, G. Cunningham, A. Gurchenko, E. Gusakov, B. Lloyd, M. O’Brien, A. Saveliev, A. Surkov, F. Volpe, and M. Walsh, Fusion Sci. Technol. 52, 202 (2007).

${ }^{23}$ E. Westerhof, S. K. Nielsen, J. W. Oosterbeek, M. Salewski, M. R. De Baar, W. A. Bongers, A. Bürger, B. A. Hennen, S. B. Korsholm, F. Leipold, D. Moseev, M. Stejner, D. J. Thoen, and TEXTOR Team, Phys. Rev. Lett. 103, 125001 (2009).

${ }^{24}$ S. K. Nielsen, P. K. Michelsen, S. K. Hansen, S. B. Korsholm, F. Leipold, J. Rasmussen, M. Salewski, M. Schubert, M. Stejner, J. Stober, D. Wagner, and ASDEX Upgrade Team, Phys. Scr. 92, 024001 (2017).

${ }^{25}$ S. K. Hansen, S. K. Nielsen, M. Salewski, M. Stejner, J. Stober, and ASDEX Upgrade Team, EPJ Web Conf. 149, 03020 (2017).

${ }^{26}$ S. K. Hansen, S. K. Nielsen, M. Salewski, M. Stejner, J. Stober, and ASDEX Upgrade Team, Plasma Phys. Controlled Fusion 59, 105006 (2017).

${ }^{27}$ R. Wilhelm, V. Erckmann, G. Janzen, W. Kasparek, G. Müller, E. Räuchle, P. G. Schüller, K. Schwörer, M. Thumm, and W VII-A Team, Plasma Phys. Controlled Fusion 26, 1433 (1984).

${ }^{28}$ H. P. Laqua, V. Erckmann, H. J. Hartfuß, H. Laqua, W7-AS Team, and ECRH Group, Phys. Rev. Lett. 78, 3467 (1997).

${ }^{29}$ H. P. Laqua, Plasma Phys. Controlled Fusion 49, R1 (2007).

${ }^{30}$ A. Köhn, G. Birkenmeier, A. Chusov, P. Diez, A. Feuer, U. Höfel, H. Höhnle, E. Holzhauer, W. Kasparek, S. Merli, M. Ramisch, J. Seifert, S. Wolf, and U. Stroth, Plasma Phys. Controlled Fusion 55, 014010 (2013).

${ }^{31}$ S. Kubo, M. Nishiura, K. Tanaka, D. Moseev, S. Ogasawara, T. Shimozuma, Y. Yoshimura, H. Igami, H. Takahashi, T. I. Tsujimura, R. Makino, and LHD Experiment Group, J. Instrum. 11, C06005 (2016).

${ }^{32}$ A. Fasoli, S. Brunner, W. A. Cooper, J. P. Graves, P. Ricci, O. Sauter, and L. Villard, Nat. Phys. 12, 411 (2016).

${ }^{33}$ E. Z. Gusakov and A. Y. Popov, Phys. Plasmas 23, 082503 (2016).

${ }^{34}$ R. B. White and F. F. Chen, Plasma Phys. 16, 565 (1974).

${ }^{35} \mathrm{M}$. Porkolab, in Proceedings of 2 nd Workshop Hot Electron Ring Physics (San Diego), edited by N. A. Uckan (National Technical Information Service, Alexandria, 1982), Vol. 1, p. 237.

${ }^{36}$ E. Z. Gusakov and A. V. Surkov, Plasma Phys. Controlled Fusion 49, 631 (2007).

37I. Y. Dodin and A. V. Arefiev, Phys. Plasmas 24, 032119 (2017).

${ }^{38}$ A. V. Arefiev, I. Y. Dodin, A. Köhn, E. J. Du Toit, E. Holzhauer, V. F. Shevchenko, and R. G. L. Vann, Nucl. Fusion 57, 116024 (2017).

${ }^{39}$ N. I. Budko and V. V. Vaskov, Geomagn. Aeron. 34, 350 (1994), see http:// eos.wdcb.ru/transl/gma/9403/pap15.ps.

${ }^{40}$ R. Yan, C. Ren, J. Li, A. V. Maximov, W. B. Mori, Z.-M. Sheng, and F. S. Tsung, Phys. Rev. Lett. 108, 175002 (2012).

${ }^{41}$ V. Shevchenko, M. R. O’Brien, D. Taylor, A. N. Saveliev, and MAST Team, Nucl. Fusion 50, 022004 (2010). 
${ }^{42}$ V. F. Shevchenko, Y. F. Baranov, T. Bigelow, J. B. Caughman, S. Diem, C. Dukes, P. Finburg, J. Hawes, C. Gurl, J. Griffiths, J. Mailloux, M. Peng, A. N. Saveliev, Y. Takase, H. Tanaka, and G. Taylor, EPJ Web Conf. 87, 02007 (2015).

${ }^{43}$ N. A. Lopez and F. M. Poli, Plasma Phys. Controlled Fusion 60, 065007 (2018).

${ }^{44}$ A. T. Lin and C.-C. Lin, Phys. Rev. Lett. 47, 98 (1981).

${ }^{45}$ M. G. Senstius, S. K. Nielsen, and R. G. L. Vann, EPJ Web Conf. 203, 01010 (2019).

${ }^{46}$ D. Wagner, G. Grünwald, F. Leuterer, A. Manini, F. Monaco, M. Münich, H. Schütz, J. Stober, H. Zohm, T. Franke, M. Thumm, G. Gantenbein, R. Heidinger, A. Meier, W. Kasparek, C. Lechte, A. Litvak, G. G. Denisov, A. V. Chirkov, E. M. Tai, L. G. Popov, V. O. Nichiporenko, V. E. Myasnikov, E. A Solyanova, S. A. Malygin, F. Meo, and P. Woskov, Nucl. Fusion 48, 054006 (2008).
${ }^{47}$ M. Stejner, S. Nielsen, A. S. Jacobsen, S. B. Korsholm, F. Leipold, F. Meo, P. K. Michelsen, D. Moseev, J. Rasmussen, M. Salewski, M. Schubert, J. Stober, D. H. Wagner, and ASDEX Upgrade Team, Rev. Sci. Instrum. 85, 093504 (2014).

${ }^{48}$ J. Ongena, R. Koch, R. Wolf, and H. Zohm, Nat. Phys. 12, 398 (2016).

${ }^{49}$ A. D. Piliya, in Proceedings of 10th International Conference on Phenomena in Ionized Gases (Oxford), edited by R. N. Franklin (Donald Parsons \& Co. Ltd., Oxford, 1971) p. 320.

${ }^{50}$ M. N. Rosenbluth, Phys. Rev. Lett. 29, 565 (1972).

${ }^{51}$ A. D. Piliya, Sov. Phys.-JETP 37, 629 (1973), http://www.jetp.ac.ru/cgi-bin/dn/ e_037_04_0629.pdf.

${ }^{52} \mathrm{H}$. Bindslev, J. Atmos. Terr. Phys. 58, 983 (1996).

${ }^{53}$ R. Ochoukov, V. Bobkov, C. Bruhn, R. D’Inca, H. Faugel, H. Fuenfgelder, R. McDermott, P. Schneider, G. Suarez-Lopez, J.-M. Noterdaeme, ASDEX Upgrade Team, and MST1 Team, EPJ Web Conf. 157, 03038 (2017).

${ }^{54}$ D. G. Swanson, Plasma Waves, 2nd ed. (IOP Publishing, Bristol, 2003). 